\title{
REVIEW
}

\section{A review on the essential oil chemical profile of Salvia genus from Iran}

Dinous Asgarpanah*

Department of Pharmacognosy, Faculty of Pharmacy and Pharmaceutical Sciences, Tehran Medical Sciences, Islamic Azad University, Tehran, IRAN

*Corresponding author. Email: taxolfa@yahoo.com

Submitted: 03.01.2021; Accepted: 05.07.2021

\begin{abstract}
Iran is one of the major centres of diversity for Salvia, with 58 species of which $25 \%$ are endemic. A literature-based survey of the chemical profile of Salvia species essential oil found in Iran was carried out. As a result, 46 species belonging to Salvia genus were identified for their essential oil composition. A concise review of the scientific literature pertaining to components of Salvia essential oils and volatile fractions is presented. The present review showed that the Iranian Salvia species have been classified into four main categories based on the GC/MS and GC-FID analyses of their essential oils. monoterpene chemotype, mono and sesquiterpene chemotype, sesquiterpene chemotype and GLV chemotype which stands for green leaf volatiles. It was indicated the most of the studied Salvia species were considered as sesquiterpene chemotype. Among the sesquiterpenes, $\beta$-caryophyllene followed by germacrene $D$, spathulenol and caryophyllene oxide were the most common and abundant in Persian Salvia species. The $\beta$ caryophyllene and germacrene D were reported from 27 and 22 Salvia species in Iran, respectively.
\end{abstract}

Keywords: Salvia, Lamiaceae, Essential oil

\section{Introduction}

Aromatic plants are at present widely studied for their large therapeutic potential and benefits. These benefits depend largely on essential oils which, in general terms, occur in many herbs. Essential oils are used to give flavour to foods and drinks and as fragrances in the food and cosmetics industries, where numerous herbal plant and spice ingredients are components in the manufacture of skin creams, lip balms, shampoos, soaps and perfumes (de Almeida et al., 2011).

The largest genus of the Lamiaceae family, the genus Salvia L. represents an enormous and cosmopolitan assemblage of nearly 1000 species displaying remarkable variation. It has undergone marked species radiations in three regions of the world: Central and South America (500 spp.), Central Asia/ Mediterranean (250 spp.) and Eastern Asia (90 spp.) (Walker et al., 2004, Claßen-Bockhoff, 2017). Iran, particularly, is one of the centers of origin of the genus with 58 species, here called with the common Persian name of "MaryamGoli" and about $25 \%$ of endemics (Mozaffarian, 1996.)

Several species of Salvia are cultivated for their aromatic characteristics and are used as flavourings, food condiments, cosmetics, and perfume additives (Firdous et al., 1999). Additionally, Salvia species have commonly been widely used as folk medicines as antibacterial, antiviral, antitumor, spasmolytic, antioxidant and anti-inflammatory treatments and have further been used in the treatment of nervous and gastrointestinal conditions (Lu \& Foo, 2002; Tepe et al., 2004)

The diversity, species richness, and variation, as well as chemical properties have led to much research on the genus Salvia. Diterpenoids of the abietane, icetexane, labdane, neoclerodane and phenalenone types (Kusumi et al., 1985; Nieto et al., 2000), triterpenes and sterols (Rauter et al., 2007), anthocyanins, coumarins, polysaccharides, flavonoids and phenolic acids and their derivatives (Lu \& Foo, 2002) were reported as major 
constituents of Salvia species. Most Salvia species are rich in essential oils, and various biologically active monoterpene/sesquiterpene have been reported in them possessing diverse biological activities such as antioxidant (Tepe et al., 2004; Kabouche et al., 2007; Guy et al., 2010), anti-inflammatory [11,12], analgesic and antipyretic [13], antiepileptic, anti-ulcerogenic, tranquillizing activities [14] and (Guy et al., 2010; ElSayed et al., 2006) antimicrobial activities (Tepe et al., 2004; Tepe et al., 2005; Usama \& Mohamed, 2006). Furthermore, the Salvia species, often pleasantly aromatic plants of potential economic interest, comprise most of the essential oil rich genera of the Lamiaceae, and particularly tend to accumulate monoterpenoidrich essential oils.

There are several reports on the chemical composition of the essential oils from the members of the genus Salvia found in Iran. With the purpose of giving an overview of the structural complexity and interesting chemical diversity of the essential oil composition of the genus Salvia, here we review systematically the articles reported over the past decades, concerning the isolation and structural elucidation of Persian Salvia sp. essential oil components. Particularly, we report on the essential oil composition referring to 46 native and endemic species of Salvia grow wild in Iran.

\section{Materials and Methods}

The present study was carried out based on the literature review of the essential oil chemical composition of native or endemic Salvia species found in Iran. The data presented in this work was collected using scientific data retrieved from encyclopaedia books, journals, articles, and websites including Pubmed, Scopus and Google Scholar.

\section{Results and Discussion}

In this review the essential oil chemical profile of 46 species including the native and endemic Salvia species found in Iran have been reported. All the native and endemic species which grow wild in Iran are given in Table 1.

Table 1. The native and endemic Salvia species found in Iran (Mozaffarian, 1996).

\begin{tabular}{|c|c|c|c|c|c|}
\hline No. & Scientific Name & Persian Name & Synonyms & $\mathbf{N} / \mathbf{E}$ & [Ref.] \\
\hline 1 & S. aegyptiaca L. & Mesri Maryam-goli & S. multicaulis Vahl. & Native & (Jassbi et al., 2012) \\
\hline 2 & S. aethiopis L. & Pashmalu Maryam-goli & S. sclarea L. & Native & $\begin{array}{l}\text { (Rajabi et al., 2014; Salimpour et } \\
\text { al., 2011) }\end{array}$ \\
\hline 3 & S. aristata Aucher ex Benth. & Sikhak-dar Maryam-goli & - & Native & (Emadipoor et al., 2016) \\
\hline 4 & S. artopatana Bunge & Azerbayjani Maryam-goli & - & Native & $\begin{array}{l}\text { (Jassbi et al., 2012; Salimpour et } \\
\text { al., 2011; Mirza \& Ahmadi, 2000) }\end{array}$ \\
\hline 5 & S. bazmanica Rech. f. \& Esfand & Bazmani Maryam-goli & S. multicaulis Vahl. & Endemic & (Akhgar et al., 2011) \\
\hline 6 & S. brachyantha (Bordz.) Pobed & Forutan Maryam-goli & S. indica $\mathrm{L}$. & Native & (Jamzad et al., 2012) \\
\hline 7 & S. bracteata Banks \& Soland. & Barge-dar Maryam-goli & - & Native & $\begin{array}{l}\text { (Amiri, 2077; Sefidkon et al., } \\
\text { 2007) }\end{array}$ \\
\hline 8 & S. ceratophylla L. & $\begin{array}{l}\text { Shakh-gavazni Maryam- } \\
\text { goli }\end{array}$ & - & Native & - \\
\hline 9 & S. choloroleuca Rech. f. \& Aell. & Sefid Maryam-goli & - & Native & $\begin{array}{l}\text { (Jassbi et al., 2012; Khalilzadeh et } \\
\text { al., 2011) }\end{array}$ \\
\hline 10 & S. chorassanica Bunge. & Khorassani Maryam-goli & $\begin{array}{l}\text { S. limbata C. A. } \\
\text { Mey., S. splendens } \\
\text { Ker. Gawl. }\end{array}$ & Endemic & (Ebrahimi et al., 2014) \\
\hline 11 & S. compressa Vent. & Marmarashk & $\begin{array}{l}\text { S. macrosiphon } \\
\text { Boiss., S. spinosa L., } \\
\text { S. hydrangea DC. ex } \\
\text { Benth. }\end{array}$ & Native & (Mirza \& Bahernik, 2007) \\
\hline
\end{tabular}


S. frigida Boiss.

S. glutinosa L.

S. grossheimii Sosn.

S. hydrangea DC.

S. hypochionaea Boiss.

S. hypoleuca Benth

S. indica L.

S. jamzadii Mozaff.

S. kermanshahensis Rech. $\mathrm{f}$.

S. lanchnocalyx Hedge.

S. lanigera Poir

S. leriifolia Benth.

S. limbata C. A. Mey.

S. macilenta Boiss.

S. macrochlamys Boiss. \& Kotschy

28

S. macrosiphon Boiss.

S. mirzayanii Rech. f. \& Esfand.

Morpojo

Arghavani Maryam-goli

Mazrae-rui Maryam-goli

Daruee Maryam-goli

Rudbari Maryam-goli

Kutah-sonbol Maryam-goli

Felestini Maryam-goli

Perspolisi Maryam-goli

Baluchestani Maryam-goli

Fenjani Maryam-goli

Isfahani Maryam-goli

Taftani Maryam-goli

Halabi Maryam-goli
Endemic

(Jassbi et al., 2012; Rajabi et al., 2014; Salehi et al., 2014)

Native

Native

Native

Native

Endemic

Endemic

, 2012. Sonbili et al. 2016; Nichavar et al., 2005)

Native

(Jamzad et al., 2011; Bahernik \& Mirza, 2010)

Endemic

Endemic

Bunge., S. staminea

Montbr. \& Auch. ex Benth.

Endemic

Native

Native

Attaran et al., 2016; Monfared \& Ghorbanli, 2010; Yousefi et al., 2015)

Native

Sajjadi \& Shahpiri, 2004; Rajabi et al., 2014; Rustaiyan et al., 2005; Morteza-Semnani et al., 2014; Mirza et al., 2005)

Native (Sonbili et al., 2005)

Native (Kazemizadeh et al., 2010)

Native (Jassbi et al., 2012; Rajabi et al., 2014; Mirza et al., 2005; Sefidkon et al., 2013; Kariminik et al., 2019; Salimpour et al., 2011)

\section{S. brachyantha}

Endemic

(Sonboli et al., 2006; Yamini et al., 2008; Mirza et al., 2003; Asadipour et al., 2013; Haghighat et al., 2012; Javidnia et al., 2002)

Native (Ahmadi \& Mirza, 1999)

Native (Rajabi et al., 2014; Mirza \& Sefidkon, 1999)

Native (Kazemi et al., 2015; Alizadeh \& Shaabani, 2012)

Endemic (Salimpour et al., 2011)

Native (Amiri, 2007; Shakeri et al., 2018)

Native (Rustaiyan et al., 2005; Salehi et al., 2005)

\section{S. aristata Aucher.} ex Benth.

Endemic

Native

Native

Native

(Jassbi et al., 2012; Rajabi et al. 2014; Mirza \& Sefidkon, 1999; Batooli et al., 2013; Salimpour et al., 2011; Lari Yazdi et al., 2005)

Native (Rustayian et al., 2005; Sajjadi \& Ghanndi, 2005; Habibi et al., 2008) 


\begin{tabular}{|c|c|c|c|c|c|}
\hline 42 & S. sahendica Boiss. \& Buhse. & Sahandi Maryam-goli & - & Endemic & (Salehi et al., 2004) \\
\hline 43 & S. santolinifolia Boiss. & Khaliji Maryam-goli & - & Native & $\begin{array}{l}\text { (Jassbi et al., 2012; Sonboli et al., } \\
\text { 2006; Rustayian et al., 2005; } \\
\text { Bahadori et al., 2016; Salehi et al., } \\
2014 \text { ) }\end{array}$ \\
\hline 44 & S. sclarea L. & Maryam-goli & - & Native & $\begin{array}{l}\text { (Rajabi et al., 2014; Batooli et al., } \\
\text { 2013; Salimpour et al., 2011) }\end{array}$ \\
\hline 45 & S. sclareopsis Bornm. ex Hedge. & Karandi Maryam-goli & S. ceratophylla L. & Endemic & (Hemmati et al., 2018) \\
\hline 46 & S. sharifii Rech. f. \& Esfand. & Jonubi Maryam-goli & $\begin{array}{l}\text { S. hydrangea DC. ex } \\
\text { Benth. }\end{array}$ & Endemic & $\begin{array}{l}\text { (Zare \& Jassbi, 2014; Asgarpanah } \\
\text { et al., 2017) }\end{array}$ \\
\hline 47 & S. spinosa L. & Khar-dar Maryam-goli & - & Native & $\begin{array}{l}\text { (Salehi et al., 2014; Bahernik \& } \\
\text { Mirza, 2005) }\end{array}$ \\
\hline 48 & $\begin{array}{l}\text { S. staminea Montbr. \& Auch. } \\
\text { ex Benth. }\end{array}$ & $\begin{array}{l}\text { Parcham-boland Maryam- } \\
\text { goli }\end{array}$ & - & Native & (Salehi et al., 2013) \\
\hline 49 & $\begin{array}{l}\text { S. suffruticosa Montbr. \& Auch. } \\
\text { ex Benth. }\end{array}$ & Bute-e Maryam-goli & $\begin{array}{l}\text { S. aristata Aucher. } \\
\text { ex Benth. }\end{array}$ & Native & $\begin{array}{l}\text { (Jamzad et al., 2011; Norouzi- } \\
\text { Arasi et al., 2005) }\end{array}$ \\
\hline 50 & S. syriaca L. & Suri Maryam-goli & - & Native & $\begin{array}{l}\text { (Lari Yazdi et al., 2005; Sefidkon \& } \\
\text { Mirza, 1999) }\end{array}$ \\
\hline 51 & S. tebesana Bunge. & Tabasi Maryam-goli & - & Native & (Goldansaz et al., 2017) \\
\hline 52 & S. trichoclada Benth. & Shakhe-korki Maryam-goli & - & Native & - \\
\hline 53 & S. urumiensis Bunge. & Urumie-e Maryam-goli & - & Endemic & (Khalilzadeh et al., 2011) \\
\hline 54 & S. verticillata $\mathrm{L}$. & Banafsh Maryam-goli & - & Native & (Rajabi et al., 2014) \\
\hline 55 & S. virgata Jacq. & Harz Maryam-goli & - & Native & $\begin{array}{l}\text { (Rajabi et al., 2014; Sefidkon \& } \\
\text { Mirza, 1999) }\end{array}$ \\
\hline 56 & S. viridis L. & Sabz Maryam-goli & - & Native & - \\
\hline 57 & S. wendelboi Hedge. & Sanandaji Maryam-goli & - & Endemic & - \\
\hline 58 & S. xanthocheila Boiss. ex Benth. & Alborzi Maryam-goli & - & Native & $\begin{array}{l}\text { (Khalilzadeh et al., 2011; Salehi et } \\
\text { al., 2005) }\end{array}$ \\
\hline
\end{tabular}

The essential oils of the Iranian Salvia species are the best-studied Salvia products. To the best of our knowledge, the essential oils from 46 species of sage, have been analysed by gas chromatography (GC) and gas chromatography/Mass (GC/MS) spectroscopy. Publications on Salvia essential oils clearly demonstrate that chemical polymorphism is characteristic of this genus, and the oil composition depends on variety, growing site, climatic conditions, and analysis method. However, the composition of volatile compounds is known for most of the species, few data are found in chemotaxonomic studies of this genus.

The Iranian Salvia species have been classified into four main categories based on the GC/MS and GC-FID (flame ionization detector) analyses of their essential oils (Jassbi et al., 2012).

The first category (monoterpene chemotype) includes the Salvia species which contain monoterpene-rich essential oils with pinane, $p$-menthane, isocamphane, and bornane C-skeletons. The bornane-type monoterpenes are mostly in the form of borneol or its acetate ester. $\alpha$-pinene, $\beta$-pinene, linalool and the related ester linalyl acetate, 1,8-cineole and borneol are characterized as the main chemical markers of the first category. Camphene and sabinene are the other major monoterpenes in some species in this chemotype. Most of the oils in this category contain $\alpha$ - and $\beta$-pinene as the monoterpene hydrocarbons, but the single-isomer is usually in higher concentration.

The second category (mono and sesquiterpene chemotype) consists of the Salvia species which contain almost equal quantities of monoterpenes with 2,6-dimethyl octane, pinane, $p$-menthane, and bornane Cskeleton, and sesquiterpenes with caryophyllane, aromadendrane, germacrane, or cadinane C-skeleton. $\beta$ caryophyllene, germacrene $D$, bicyclogermacrene, spathulenol, and caryophyllene oxide are identified as the major chemical markers of this category. 
The third category of the Salvia species (sesquiterpene chemotype) contains sesquiterpenes with caryophyllane-type C-skeleton as the predominant compounds, germacrane, aromadendrane, cadinane, and other sesquiterpenes as the other major constituents. The monoterpene fractions of these oils are in lower levels compared to the sesquiterpene.

The last category of the Salvia species (GLV chemotype) is rich in green leaf volatiles (GLVs; C6 alcohols, aldehydes, and their ester derivatives), aldehydes, esters and other fatty acids or low-molecular-weight acid derivatives, aromatic phenylpropanoids, and finally non-phenylpropanoid volatiles, which are synthesized from amino acid pathways (Baldwin, 2010). This group is called GLV producers.

Among the terpenes, the most abundant components determined are the sesquiterpenes that frequently appear as the main constituents and most of the studied Salvia species are considered as sesquiterpene chemotype. Sesquiterpenes are a class of terpenes that consist of three isoprene units and often have the molecular formula $\mathrm{C}_{15} \mathrm{H}_{24}$. They may be acyclic or contain rings.

The biosynthesis of the sesquitepenes is described as production of sesquiterpene precursor farnesyl diphosphate (FDP) from coupling dimethylallyl diphosphate (DMAPP) and isopentenyl diphosphate (IPP) (Degenhardt et al., 2009). Then, these precursors are taken as substrates by different sesquiterpene synthases, many of which are multi-product enzymes. Biochemical modifications such as oxidation or rearrangement produce the related sesquiterpenoids.

Among the sesquiterpenes, $\beta$-caryophyllene followed by germacrene-D, spathulenol and caryophyllene oxide are the most common and abundant in Persian Salvia species studied. The $\beta$-caryophyllene and germacrene D have been reported from 27 and 22 Salvia species in Iran, respectively. $\beta$-Caryophyllene and germacrene $D$ are representative of the sesquiterpenes derived from an initial macrocyclic intermediate formed by a $\mathrm{C}_{1}-$ $\mathrm{C}_{11}$ ring closure. These sesquiterpenes are distinguished by being directly derived from the trans FPP substrate and not requiring isomerization to the Cis conformation or nerolidyl diphosphate intermediate and in this regard these components might be considered the simplest of sesquiterpenes.

According to the Table 2, S. aethiopis, S. atropatana, S. bracteata, S. compressa, S. grossheimii, S. hypoleuca, S. indica, S. nemorosa, S. palaestina, S. reuteriana, S. syriaca, S. verticillata, S. verbascifolia, S. virgata, and S. xanthocheilacan are include the third category of the Salvia classification while S. brachysiphon, S. chloroleuca, S. hydrangea, S. hypoleuca, S. lachnocalyx, S. limbata, S. mirzayanii, and S. virgata can be classified in the second category of mono and sesquiterpene chemotype in which the Salvia species produce essential oils with almost equal quantities of mono and sesquiterpenes.

$\alpha$-Pinene constituted the most abundant compound in the oils of the Salvia species in the first category of monoterpene chemotype.

Salvia species, which the essential oils contain monoterpenes include $S$. aegyptiaca, S. brachycalyx, $S$. bracteata, S. eremophila, S. limbata, S. leriifolia, S. macilenta, S. mirzayanii, S. multicaulis, S. rhytidea, S. sahendica, S. santolinifolia, and S. suffruticosa.

S. atropatana, S. macrosiphon, S. persepolitana, S. reuteriana, S. sclareopsis, and S. spinosa are the species which the essential oils are rich in GLVs and other low-molecular-weight acid derivatives. Greenleaf volatiles are biosynthesized via different pathway. They are synthesized from fatty acid hydroperoxides (Dewick, 1997). 
Table 2. Components of the essential oil of Salvia species found in Iran ( $5 \%<$ ).

\begin{tabular}{|c|c|c|c|c|}
\hline $\begin{array}{l}\mathbf{N} \\
\mathbf{0}\end{array}$ & Compound & Species & $\begin{array}{l}\text { Percentage } \\
(\%)\end{array}$ & References \\
\hline 1 & $\alpha$-Thujene & S. bazmanica & 9.5 & (Akhgar et al., 2011) \\
\hline \multirow[t]{21}{*}{2} & $\alpha$-Pinene & S. aegyptiaca & 8.3 & (Jassbi et al., 2012) \\
\hline & & S. aristata & 6.7 & (Emadipour et al., 2016) \\
\hline & & S. bazmanica & 8.9 & (Akhgar et al., 2011) \\
\hline & & S. bracteata & 28.9 & (Amiri, 2007) \\
\hline & & S. chloroleuca & 11.4 & (Khalilzadeh et al., 2011) \\
\hline & & S. eremophila & 31.5 & (Jassbi et al., 2012) \\
\hline & & S. hydrangea & 5.5 & (Sonboli et al., 2006) \\
\hline & & S. hypoleuca & $5.8,29.3,5.9$ & $\begin{array}{l}\text { (Jassbi et al., 2012; Sonboli et al., 2016; } \\
\text { Nickavar et al., 2005) }\end{array}$ \\
\hline & & S. indica & 17.0 & (Jamzad et al., 2011) \\
\hline & & S. leriifolia & $15.1,16.5$ & $\begin{array}{l}\text { (Attaran et al., 2016; Monfared \& Ghorbanli, } \\
\text { 2010) }\end{array}$ \\
\hline & & S. limbata & & $\begin{array}{l}\text { (Sajjadi \& Shahpiri, 2004; Rajabi et al., 2014; } \\
\text { Rustaiyan et al., 2005) }\end{array}$ \\
\hline & & S. macilenta & $15.5,17.1,23.7$ & (Sonboli et al., 2005) \\
\hline & & & & (Ahmadi \& Mirza, 1999) \\
\hline & & S. multicaulis & 60.0 & (Shakeri et al., 2018) \\
\hline & & S. pachystachys & 15.6 & (Salehi et al., 2004) \\
\hline & & S. sahendica & 8.0 & (Jassbi et al., 2012; Sonbili et al., 2006; \\
\hline & & S. santolinifolia & 14.2 & Bahadori et al., 2016) \\
\hline & & S. tebesana & $52.5,49.3,72.4$ & (Khalilzadeh et al., 2011) \\
\hline & & & & (Goldansaz et al., 2017) \\
\hline & & S. urumiensis & 7.5 & \\
\hline & & S. tebesana & 14.0 & \\
\hline \multirow[t]{6}{*}{3} & Camphene & S. eremophila & 13.7 & (Salehi et al., 2014) \\
\hline & & S. leriifolia & 10.9 & (Yousefi et al., 2015) \\
\hline & & S. pachystachys & 11.7 & (Amiri, 2007) \\
\hline & & S. santolinifolia & $8.1,7.8$ & (Rustaiyan et al., 2005; Salehi et al., 2014) \\
\hline & & & & (Jamzad et al., 2011) \\
\hline & & S. suffruticosa & 7.9 & \\
\hline \multirow[t]{4}{*}{4} & Sabinene & S. chloroleuca & 9.6 & (Khalilzadeh et al., 2011) \\
\hline & & S. limbata & $8.3,10.6,14.5$ & $\begin{array}{l}\text { (Sajjadi \& Shahpiri, 2004; Rustaiyan et al., } \\
\text { 2005; Morteza-Semnani et al., 2014) } \\
\text { (Rustayian et al., 2005; Sajjadi \& Ghannadi, } \\
\text { 2005; Habibi et al., 2008) }\end{array}$ \\
\hline & & S. rhytidea & $5.8,13.5,17.5$ & (Salehi et al., 2004) \\
\hline & & S. sahendica & 8.5 & \\
\hline
\end{tabular}




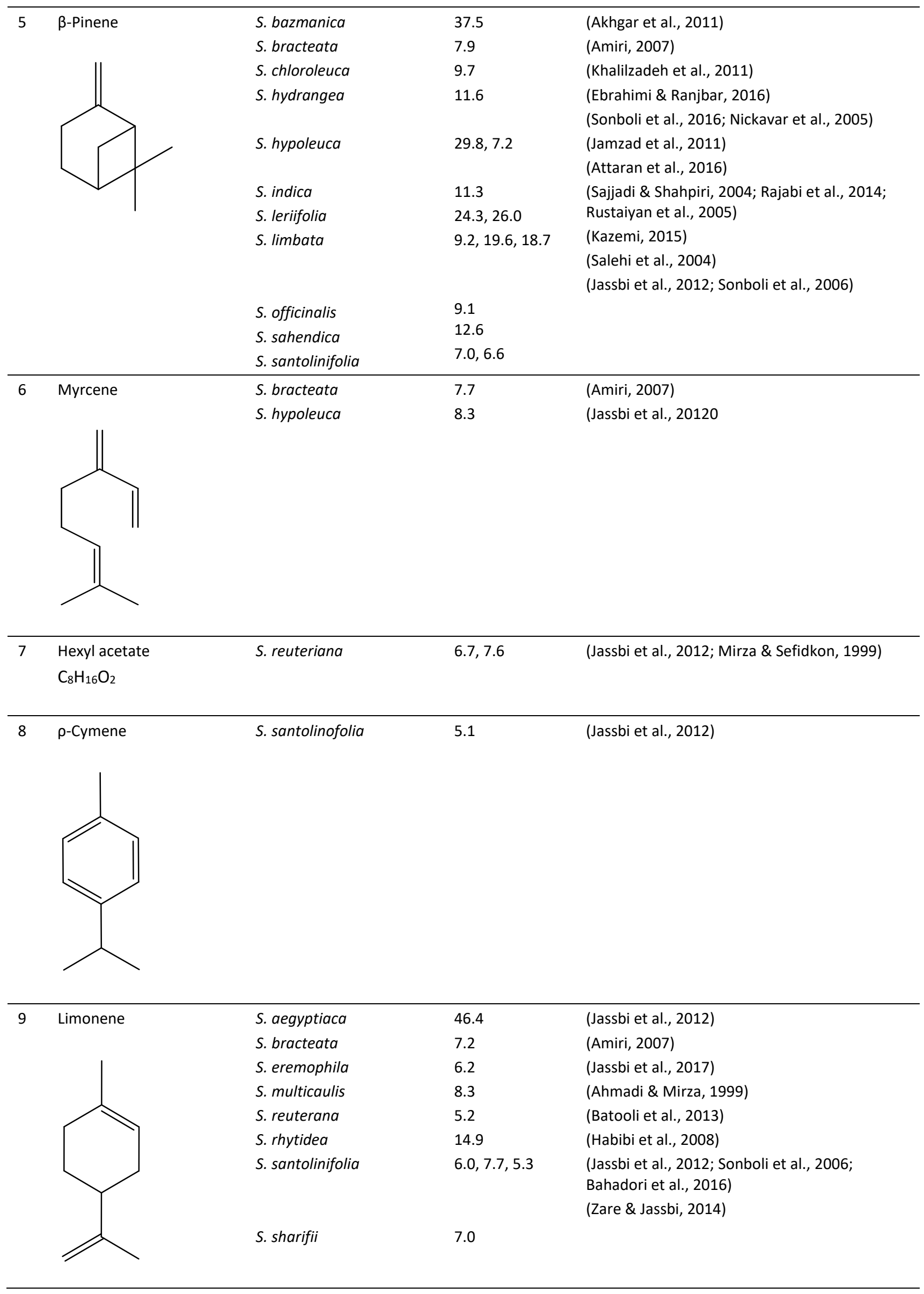




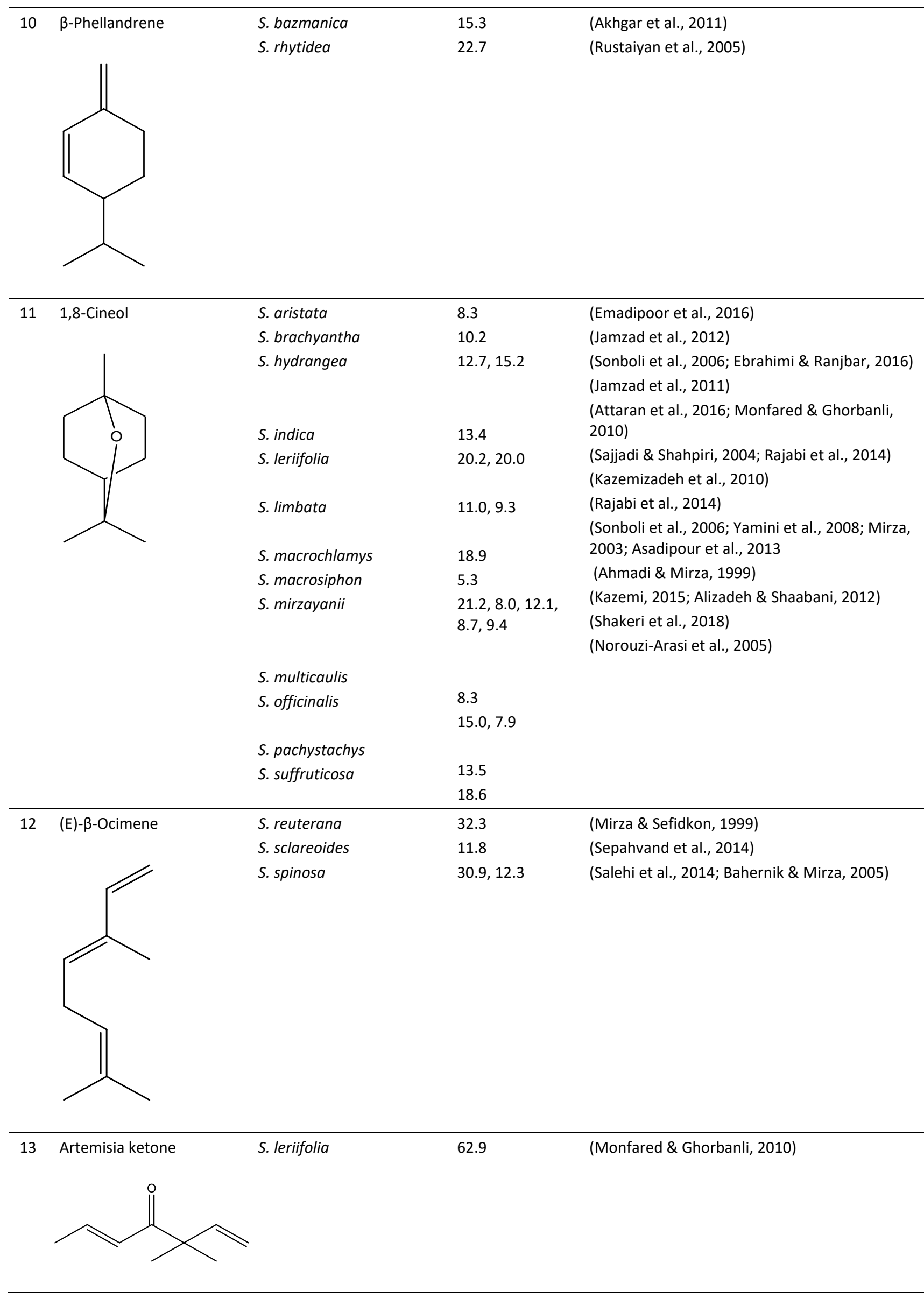




\begin{tabular}{lllll}
\hline 14 & $\alpha$-Terpinolene & S. spinosa & 32.7 & (Salehi et al., 2014)
\end{tabular}<smiles>CC1=CCC(=C(C)C)CC1</smiles>

\begin{tabular}{|c|c|c|c|c|}
\hline \multirow[t]{13}{*}{15} & \multirow[t]{13}{*}{ Linalool } & S. aethiopis & 51.6 & (Rajabi et al., 2014) \\
\hline & & S. eremophilla & $12.7-46.2$ & (Rajabi et al., 2014) \\
\hline & & S. limbata & 17.5 & (Mirza et al., 2005) \\
\hline & & S. macrosiphon & $\begin{array}{l}\text { 21.8, 34.0, 54.8, } \\
16.1-27.8\end{array}$ & $\begin{array}{l}\text { (Jassbi et al., 2012; Rajabi et al., 2014; } \\
\text { Sefidkon et al., 2013; Kariminik et al., 2019) }\end{array}$ \\
\hline & & S mirzavanii & $8.9,9.0,19.0$ & $\begin{array}{l}\text { (Sonboli et al., 2006; Yamini et al., 2008; Mirza } \\
\text { et al., 2003; Asadipour et al., 2013; Javidnia et } \\
\text { al., 2002) }\end{array}$ \\
\hline & & 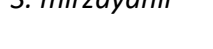 & $11.8,5.2$ & Rajabi et al., 2014) \\
\hline & & & & (Rajabi et al., 2014; Salimpour et al., 2011) \\
\hline & & & & ((Sepahvand et al., 2014) \\
\hline & & S. nemorosa & & \\
\hline & & S. sclarea & $5.0-8.1$ & \\
\hline & & & $7.4,12.2-21.4$ & \\
\hline & & S. sclareoides & & \\
\hline & & & 27.6 & \\
\hline 16 & $\alpha$-Thujon & S. officinalis & $5.7,41.5$ & (Kazemi , 2015; Alizadeh \& Shaabani, 2012) \\
\hline
\end{tabular}<smiles>CC1C(=O)CC2(C(C)C)CC1C2</smiles>

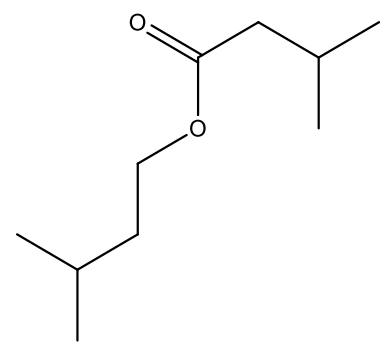




18 B-Thujone $\quad$ S. officinalis $\quad 6.8 \quad$ (Alizadeh \& Shaabani, 2012)

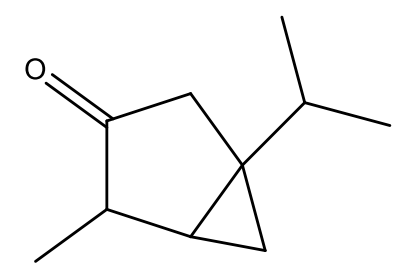

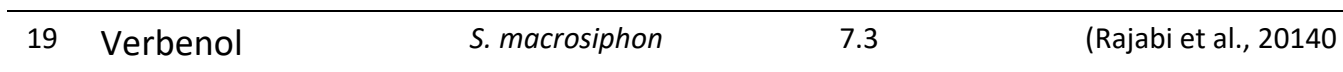<smiles>CC1=CC(O)C2CC1C2(C)C</smiles>

\begin{tabular}{|c|c|c|c|c|}
\hline \multirow[t]{7}{*}{20} & Camphor & S. hydrangea & 12.1 & (Ebrahimi \& Ranjbar, 2016) \\
\hline & & & & (Monfared \& Ghorbanli, 2010) \\
\hline & & S. leriifolia & 18.5 & (Kazemi, 2015) \\
\hline & & & & (Shakeri et al., 2018) \\
\hline & & S. officinalis & 6.0 & (Norouzi-Asadi et al., 2005) \\
\hline & & S. pachystachys & 31.0 & \\
\hline & & S. suffruticosa & 48.5 & \\
\hline
\end{tabular}

\begin{tabular}{llll}
\hline S. compressa & 9.3 & $\begin{array}{l}\text { (Mirza \& Bahernik et al., 2007) } \\
\text { (Jassbi et al., 2012) } \\
\text { (Sonboli et al., 2006) } \\
\text { (Kazemi, 2015; Alizadeh \& Shaabani, 2012) } \\
\text { (Jassbi et al., 2012) }\end{array}$ \\
$\begin{array}{l}\text { S. eremophila } \\
\text { S. hydrangea } \\
\text { S. officinalis }\end{array}$ & 22.9 & 5.2 & $12.7,8.3$ \\
S. santolinifolia & 5.2 &
\end{tabular}

\begin{tabular}{|c|c|c|c|c|}
\hline \multirow[t]{2}{*}{22} & 4-Terpineol & S. limbata & 8.9 & $\begin{array}{l}\text { (Morteza-Semnani et al., 2014) } \\
\text { (Sajjadi \& Ghannadi, 2005) }\end{array}$ \\
\hline & & S. rhytidea & 5.5 & \\
\hline
\end{tabular}




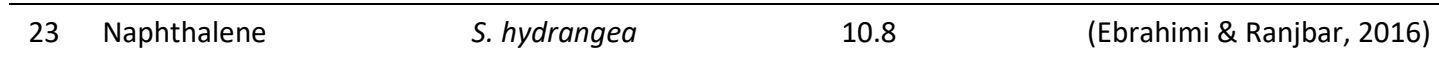<smiles>c1ccc2ccccc2c1</smiles>

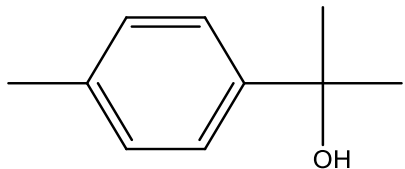

\begin{tabular}{|c|c|c|c|c|}
\hline \multirow[t]{2}{*}{25} & $\alpha$-Terpineol & S. mirzayanii & 6.3 & (Asadipour et al., 2013) \\
\hline & & S. sclarea & 5.6 & (Rajabi et al., 2014) \\
\hline
\end{tabular}<smiles>CC1=CCC(C(C)(C)O)CC1</smiles>

\begin{tabular}{|c|c|}
\hline Decanal & S. palaestina \\
\hline
\end{tabular}

27 Pulegone $\quad$ S. rhytidea $\quad 6.4 \quad$ (Sajjadi \& Ghannadi, 2005)<smiles>CC(C)=C1CCC(C)CC1=O</smiles>

\begin{tabular}{llll}
\hline Hexyl isovalerate & S. macrosiphon & $5.8,9.4$ & $\begin{array}{l}\text { (Jassbi et al., 2012; Mirza et al., 2005) } \\
\text { (Jassbi et al., 2012) }\end{array}$ \\
$\mathrm{C}_{11} \mathrm{H}_{22} \mathrm{O}_{2}$ & & 12.1 &
\end{tabular}

S. reuteriana

12.1

29 Geraniol S. compressa $10.5 \quad$ (Mirza \& Bahernik, 2007)<smiles>CC(C)CCOC(=O)CC(C)C</smiles> 


\begin{tabular}{|c|c|c|c|c|}
\hline \multirow[t]{9}{*}{30} & \multirow[t]{9}{*}{ Linalyl acetate } & S. limbata & 16.1 & (Mirza et al., 2005) \\
\hline & & \multirow[t]{3}{*}{ S. mirzayanii } & 5.4, 7.6, 12.9, & (Sonboli et al., 2006; Yamini et al., 2008; \\
\hline & & & 11.8 & Asadipour et al., 2013) \\
\hline & & & & (Rajabi et al., 2014) \\
\hline & & \multicolumn{2}{|l|}{ S. reuterana } & (Rajabi et al., 2014; Salimpour et al., 2011) \\
\hline & & \multirow{2}{*}{ S. sclarea } & 5.0 & (Rajabi et al., 2014) \\
\hline & & & $6.8,13.1-52.6$ & \\
\hline & & \multicolumn{3}{|l|}{ S. virgata } \\
\hline & & & 5.2 & \\
\hline \multirow[t]{2}{*}{31} & Hexyl valerate & S. macrosiphon & 5.0 & (Salimpour et al., 2011) \\
\hline & \multicolumn{4}{|l|}{$\mathrm{C}_{11} \mathrm{H}_{22} \mathrm{O}_{2}$} \\
\hline \multirow[t]{8}{*}{32} & \multirow[t]{8}{*}{ Bornyl acetate } & S. chloroleuca & 5.9 & (Jassbi et al., 2012) \\
\hline & & S. eremophila & 5.4 & (Jassbi et al., 2012) \\
\hline & & S. limbata & 12.5 & (Rajabi et al., 2014) \\
\hline & & S. macrosiphon & 7.8 & (Rajabi et al., 20140 \\
\hline & & S. multicaulis & 18.1 & (Ahmadi \& Mirza, 1999) \\
\hline & & S. officinalis & 5.0 & (Kazemi, 2015) \\
\hline & & S. syriaca & 10.5 & (Lari Yazdi et al., 2005) \\
\hline & & S. urumiensis & 7.7 & (Khalilzadeh et al., 2011) \\
\hline 33 & Thymol & S. macilenta & 5.2 & (Sonboli et al., 2005) \\
\hline
\end{tabular}<smiles>Cc1ccc(C(C)C)c(O)c1</smiles><smiles>Cc1ccc(C(C)C)cc1O</smiles><smiles>C=CC1(C)CCC(C(C)C)=CC1C(=C)C</smiles>

$\begin{array}{lll}\text { S. limbata } & 5.1 & \text { (Sajjadi \& Shahpiri, 2004) } \\ \text { S. syriaca } & 7.9 & \text { (Lari Yazdi et al., 2005) }\end{array}$




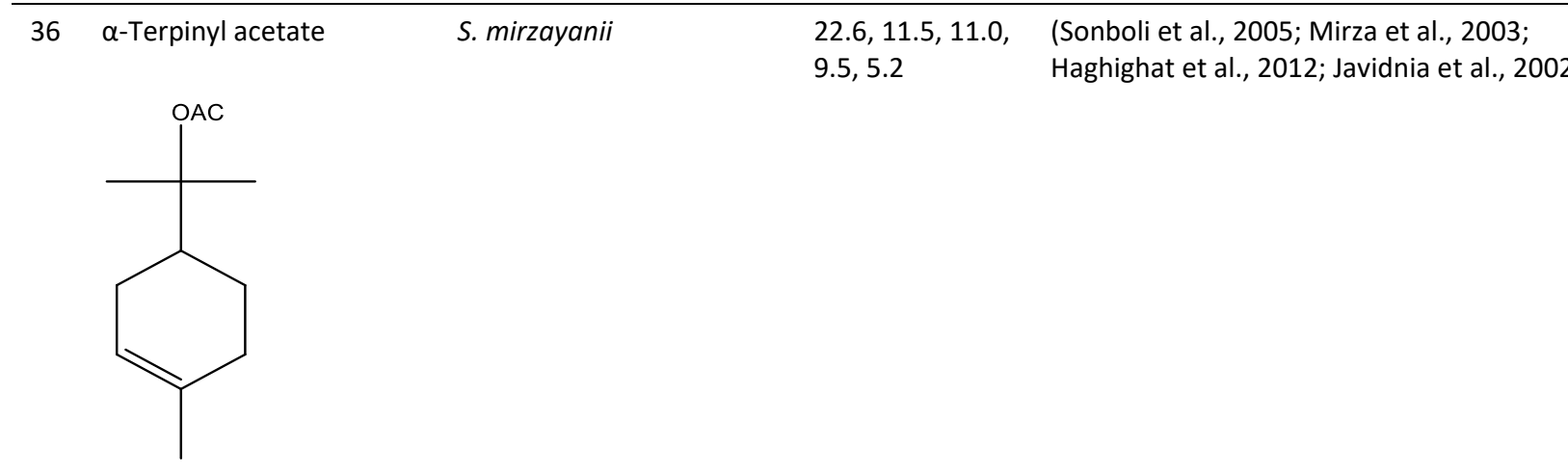

S. atropatana
S. aethiopis

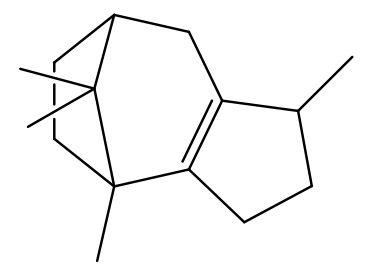

\begin{tabular}{clccr}
\hline 40 & $\begin{array}{l}\text { Hexyl hexanoate } \\
\mathrm{C}_{12} \mathrm{H}_{24} \mathrm{O}_{2}\end{array}$ & S. macrosiphon & (Jassbi et al., 2012) \\
\hline 41 & Isolongifolene & S. oligophylla & 9.9 & (Salimpour et al., 2011) \\
& & S. reuterana & 6.5 & (Salimpour et al., 2011)
\end{tabular}<smiles>CC12CCCC3(C1)C2=CCCC3(C)C</smiles> 


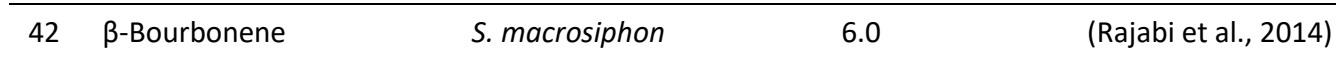

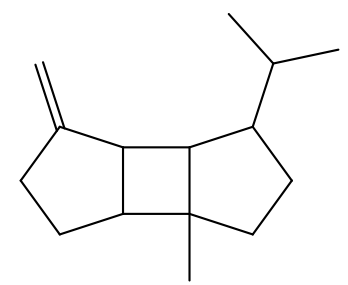

43 B-Elemene $\begin{array}{lll}\text { S. aethiopis } \\ \begin{array}{l}\text { S. macrosiphon } \\ \text { S. reuteriana }\end{array}\end{array}$

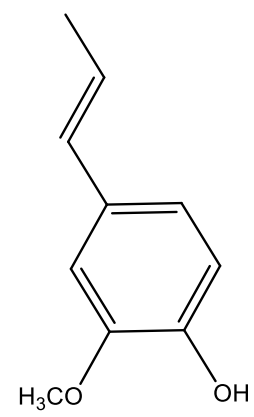

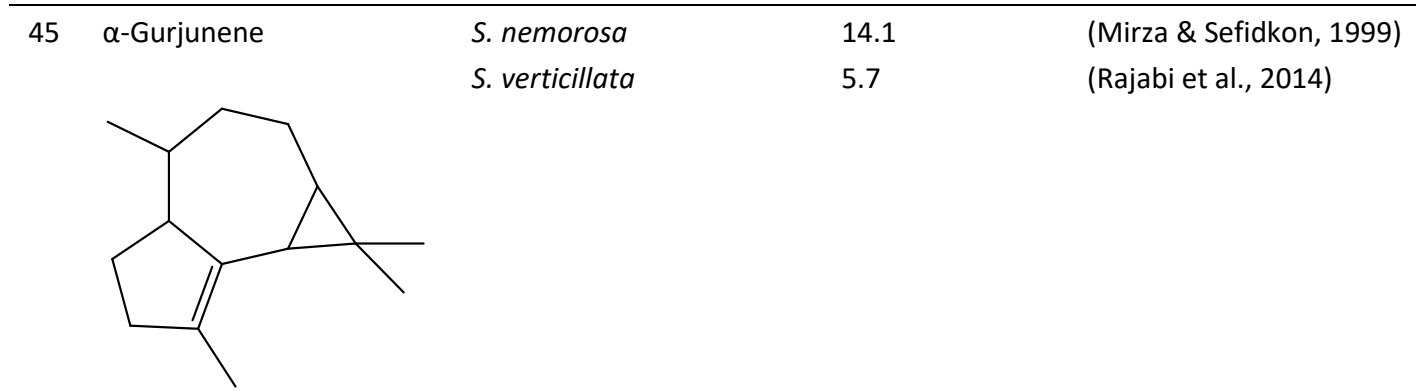

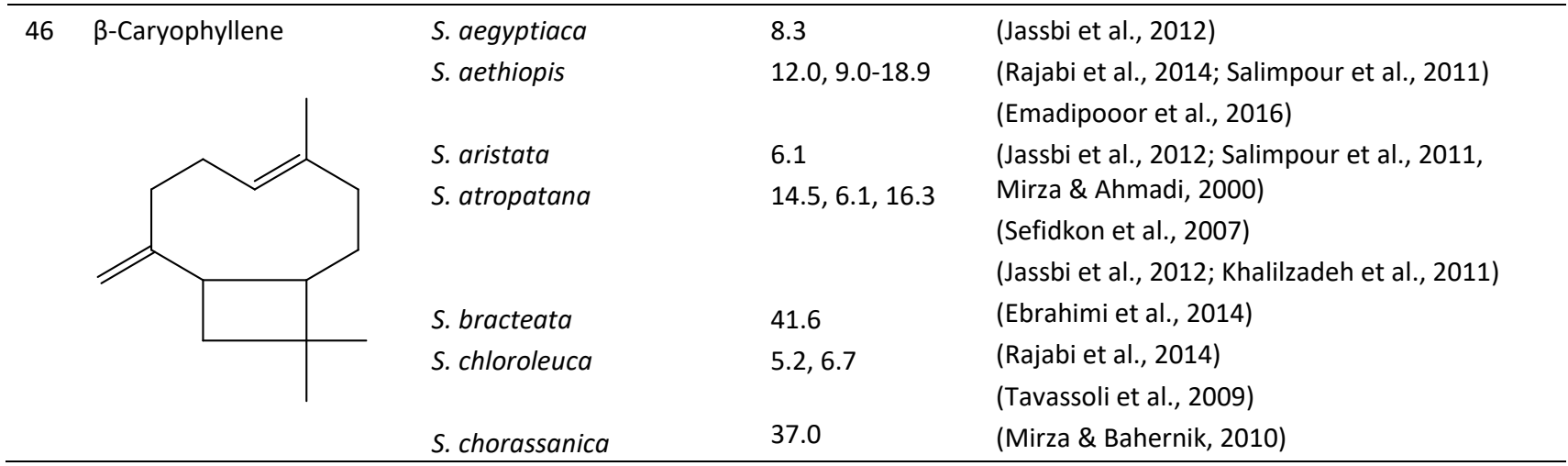




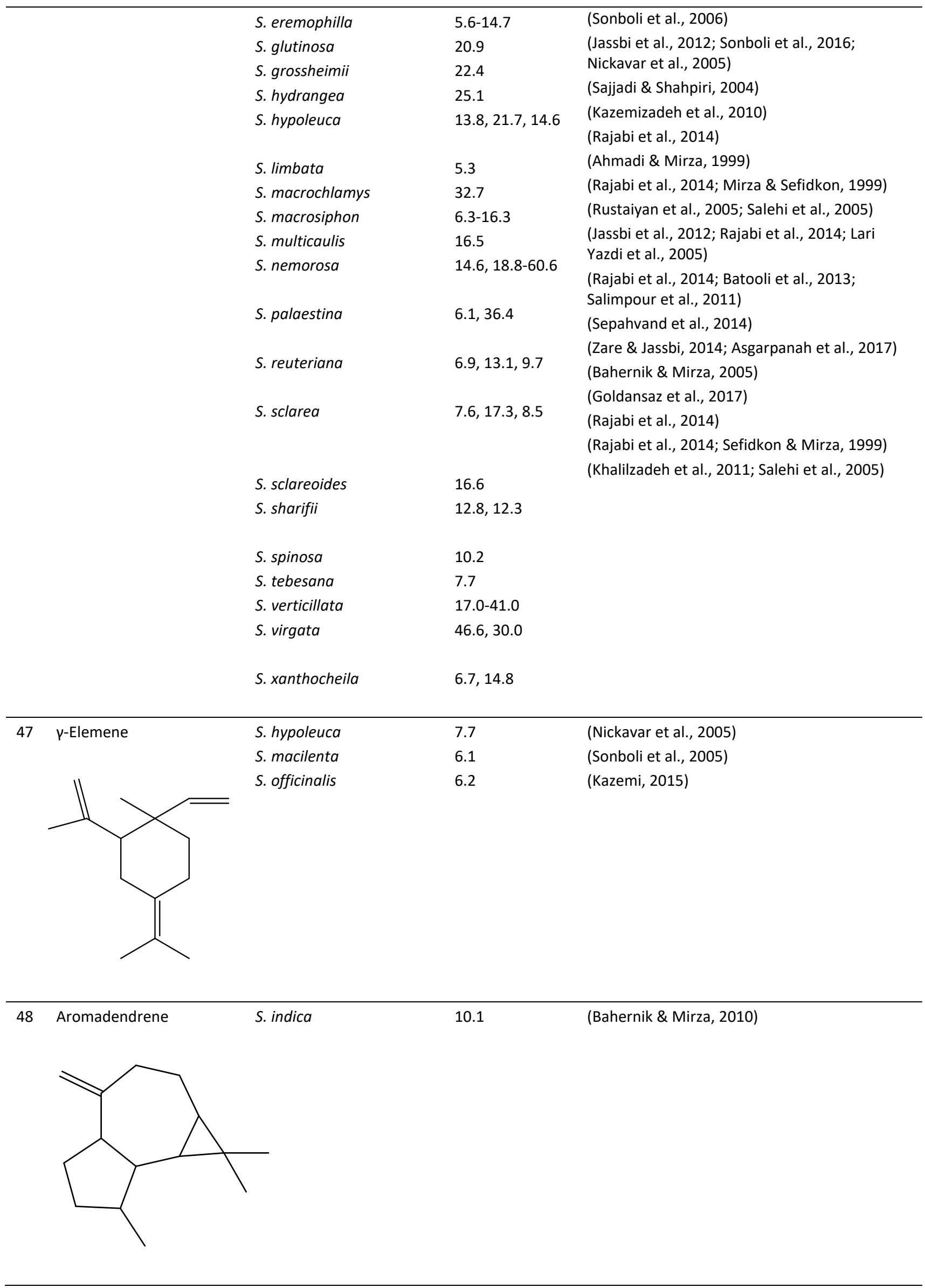



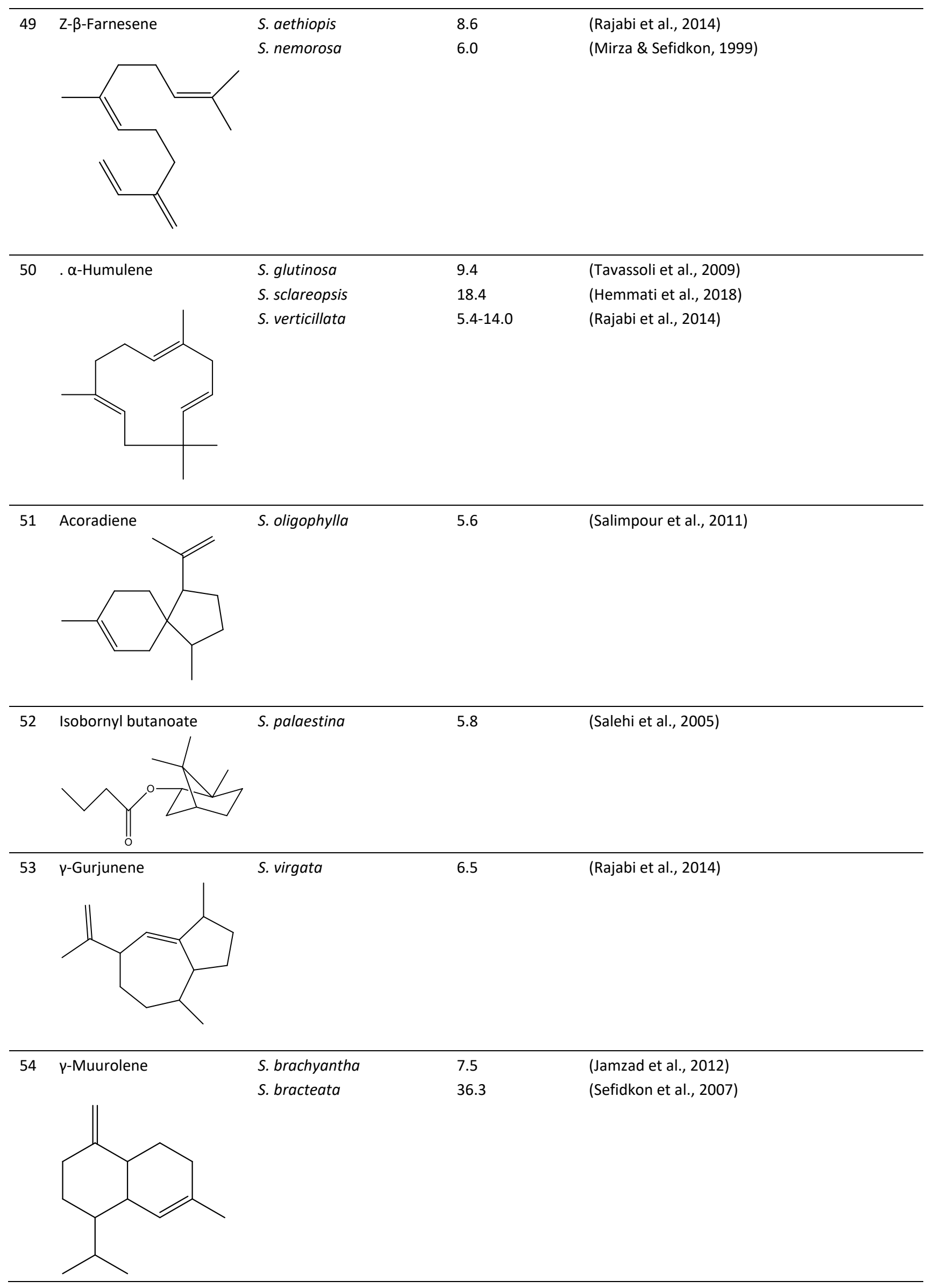


\begin{tabular}{|c|c|c|c|c|}
\hline 55 & $\alpha$-Amorphene & S. hydrangea & 9.3 & (Ebrahimi \& Ranjbar, 2016) \\
\hline \multirow[t]{28}{*}{56} & Germacrene D & S. aethiopis & $61.5,25.2$ & (Rajabi et al., 2014; Salimpour et al., 2011) \\
\hline & & & & (Jassbi et al., 2012; Salimpour et al., 2011) \\
\hline & & S. atropatana & $19.7,5.1$ & $\begin{array}{l}\text { (Jassbi et al., 2012; Khalilzadeh et al., 2011) } \\
\text { (Tavassoli et al., 2009) }\end{array}$ \\
\hline & & S. chloroleuca & $15.7,15.7$ & $\begin{array}{l}\text { (Mirza \& Bahernik, 2010) } \\
\text { (Jassbi et al., 2012) }\end{array}$ \\
\hline & & S. glutinosa & 18.0 & (Bahrnik \& Mirza, 2010) \\
\hline & & S. grossheimii & 45.4 & (Mirza et al., 2005) \\
\hline & & S. hypoleuca & 8.8 & (Rajabi et al., 2014) \\
\hline & & S. indica & 10.4 & (Rajabi et al., 2014; Mirza \& Sefidkon, 1999) \\
\hline & & S. limbata & 25.7 & (Salehi et al., 2005) \\
\hline & & S. macrosiphon & $10.5-8.1$ & (Jassbi et al., 2012; Mirza \& Sefidkon, 1999; \\
\hline & & S. nemorosa & $5.6,5.5-6.3$ & $\begin{array}{l}\text { Batooli et al., 2013; Salimpour et al., 2011) } \\
\text { (Salehi et al., 2004) }\end{array}$ \\
\hline & & S. palaestina & 14.0 & (Rajabi et al., 2012; Batooli et al., 2013; \\
\hline & & S. reuteriana & $\begin{array}{l}14.1,11.2,11.2 \\
21.2\end{array}$ & $\begin{array}{l}\text { Salimpour et al., 2011) } \\
\text { (Sepahvand et al., 2014) }\end{array}$ \\
\hline & & & & (Zare \& Jassbi, 2014; Asgarpanah et al., 2017) \\
\hline & & S. sahendica & & (Salehi et al., 2013) \\
\hline & & S. sclarea & 8.3 & (Sefidkon \& Mirza, 1999) \\
\hline & & & $\begin{array}{l}12.7,20.8,9.5- \\
17.7\end{array}$ & $\begin{array}{l}\text { (Khalilzadeh et al., 2011) } \\
\text { (Rajabi et al., 2014) }\end{array}$ \\
\hline & & S. sclareoides & & (Sefidkon \& Mirza, 1999) \\
\hline & & S. sharifii & & (Khalilzadeh et al., 2011; Salehi et al., 2005) \\
\hline & & & 10.0 & \\
\hline & & S. staminea & $9.5,30.3$ & \\
\hline & & S. syriaca & & \\
\hline & & S. urumiensis & 36.3 & \\
\hline & & S. verticillate & 29.2 & \\
\hline & & S. virgata & 5.2 & \\
\hline & & S. xanthocheila & $6.4-13.0$ & \\
\hline & & & 5.7 & \\
\hline & & & $44.0,17.6$ & \\
\hline
\end{tabular}

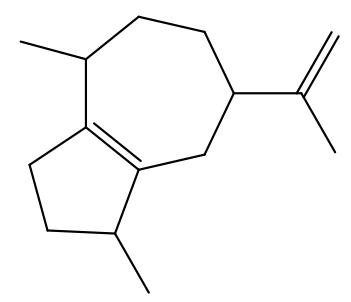




\begin{tabular}{|c|c|c|c|c|}
\hline \multirow[t]{15}{*}{58} & Bicyclogermacrene & S. aethiopis & 5.8 & (Rajabi et al., 2014) \\
\hline & & S. bracteata & 9.9 & (Sefidkon et al., 2007) \\
\hline & & S. chloroleuca & $18.5,17.0$ & (Jassbi et al., 2012; Khalilzadeh et al., 2011) \\
\hline & & & & (Mirza \& Baher, 2010) \\
\hline & & S. grossheimii & 7.1 & (Jassbi et al., 2012; Sonboli et al., 2016; \\
\hline & & S. hypoleuca & $19.8,37.3,15.3$ & $\begin{array}{l}\text { Nickavar et al., 2005) } \\
\text { (Sajjadi \&Shahpiri, 2004) }\end{array}$ \\
\hline & & & & (Haghighat et al., 2012) \\
\hline & & S. limbata & 21.1 & (Rajabi et al., 2014) \\
\hline & & S. mirzayanii & 8.0 & (Batooli et al., 2013) \\
\hline & & S. nemorosa & 7.7 & (Salehi et al., 2004) \\
\hline & & S. reuterana & 8.2 & (Batooli et al., 2013) \\
\hline & & S. sahendica & 15.8 & (Zare \& Jassbi, 2014; Asgarpanah et al., 2017) \\
\hline & & S. sclarea & 8.8 & (Rajabi et al., 2014) \\
\hline & & S. sharifii & $5.6,15.7$ & \\
\hline & & S. verticillata & $13.0-21.0$ & \\
\hline 59 & $\alpha$-Muurolene & S. sclareopsis & 8.4 & (Hemmati et al., 2018) \\
\hline
\end{tabular}<smiles>CC1=CC2C(CC1)C(C)=CCC2C(C)C</smiles>

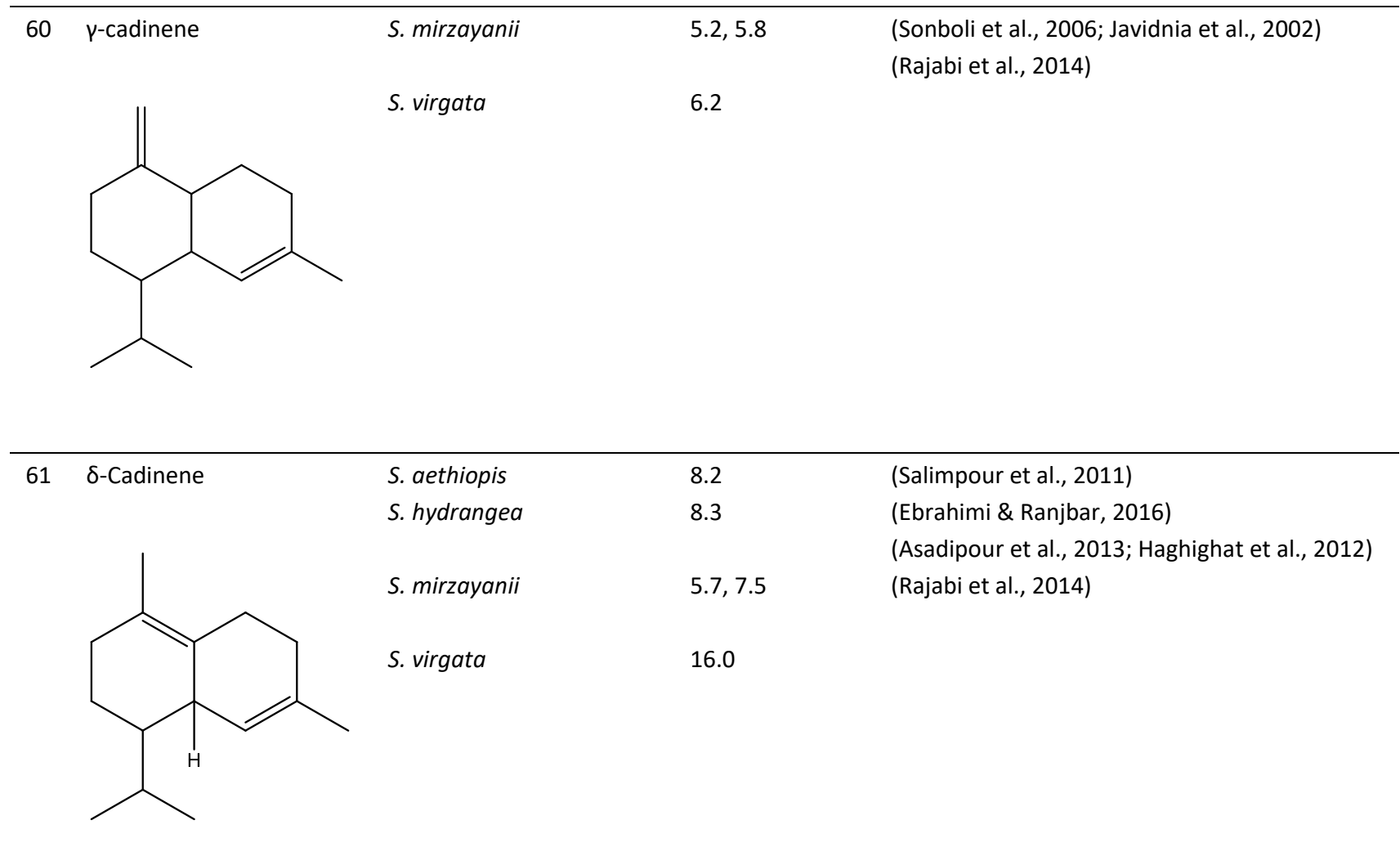




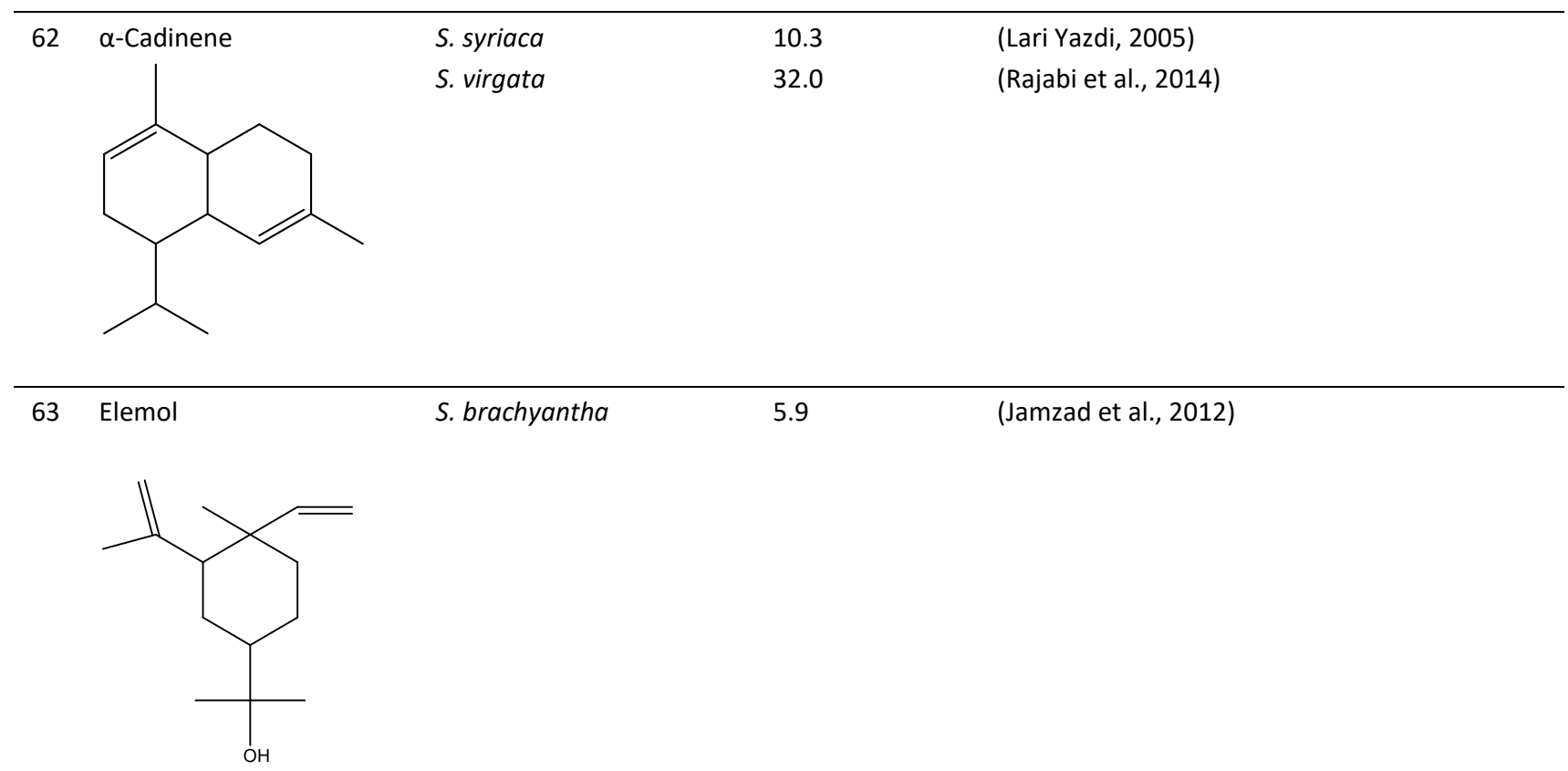<smiles>CC1=CC=CC2(C)CCC(C(C)(C)O)CC12</smiles>

65 Germacrene B $\begin{array}{lll}\text { S. atropatana } & 10.0 & \begin{array}{l}\text { (Mirza \& Ahmadi, 2000) } \\ \text { S. nemorosa } \\ \text { S syriaca } \\ \text { S. virgata }\end{array} \\ \begin{array}{l}\text { (Mirza \& Sefidkon, 1999) } \\ \text { (Sefidkon \& Mirza, 1999) } \\ \text { (Sefidkon \& Mirza, 1999) }\end{array}\end{array}$

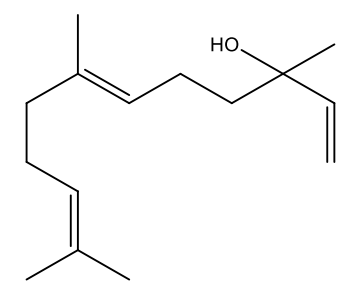

\begin{tabular}{|c|c|c|c|c|}
\hline \multirow[t]{2}{*}{67} & Hexyl octanoate & S. atropatana & 12.2 & (Mirza \& Ahmadi, 2000) \\
\hline & $\mathrm{C}_{14} \mathrm{H}_{28} \mathrm{O}_{2}$ & S. macrosiphon & 8.9 & (Sefidkon et al., 2013) \\
\hline
\end{tabular}

\begin{tabular}{llll}
\hline 68 Spathulenol & S. brachyantha & 6.2 & (Jamzad et al., 2012) \\
S. chloroleuca & 5.1 & (Khalilzadeh et al., 2011) \\
S. chorassanica & 39.5 & (Ebrahimi et al., 2014) \\
S. eremophilla & $22.0-35.4$ & (Rajabi et al., 2014) \\
\hline
\end{tabular}




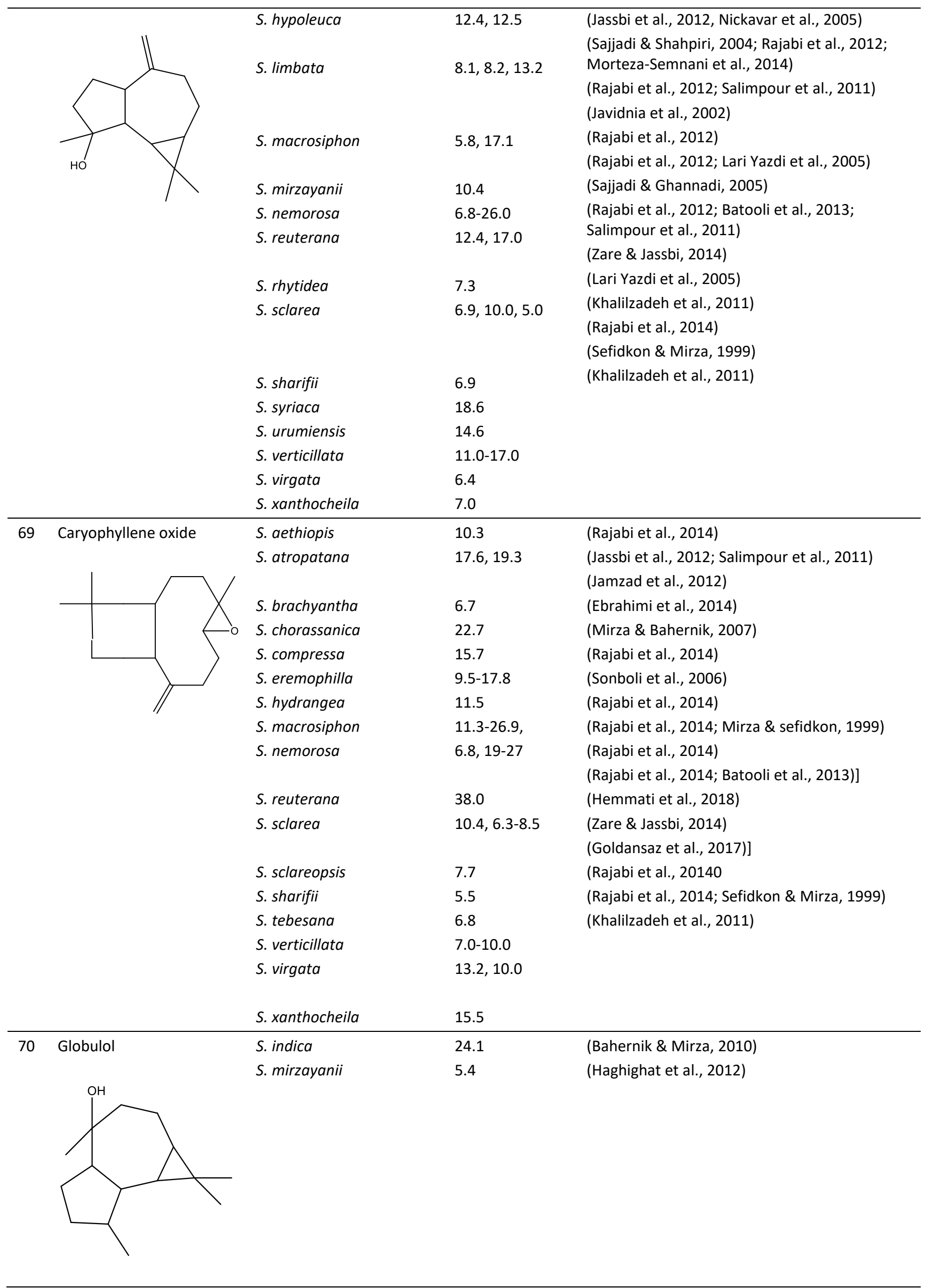




$\begin{aligned} & \text { S. hypoleuca } \\ & \text { S. officinalis }\end{aligned}$
$\begin{aligned} & \text { S. sclareopsis } \\ & \text { (Nickavar et al., 2005) }\end{aligned}$<smiles>CC1=CC2C(C(C)C)CCC(O)C2CC1</smiles>

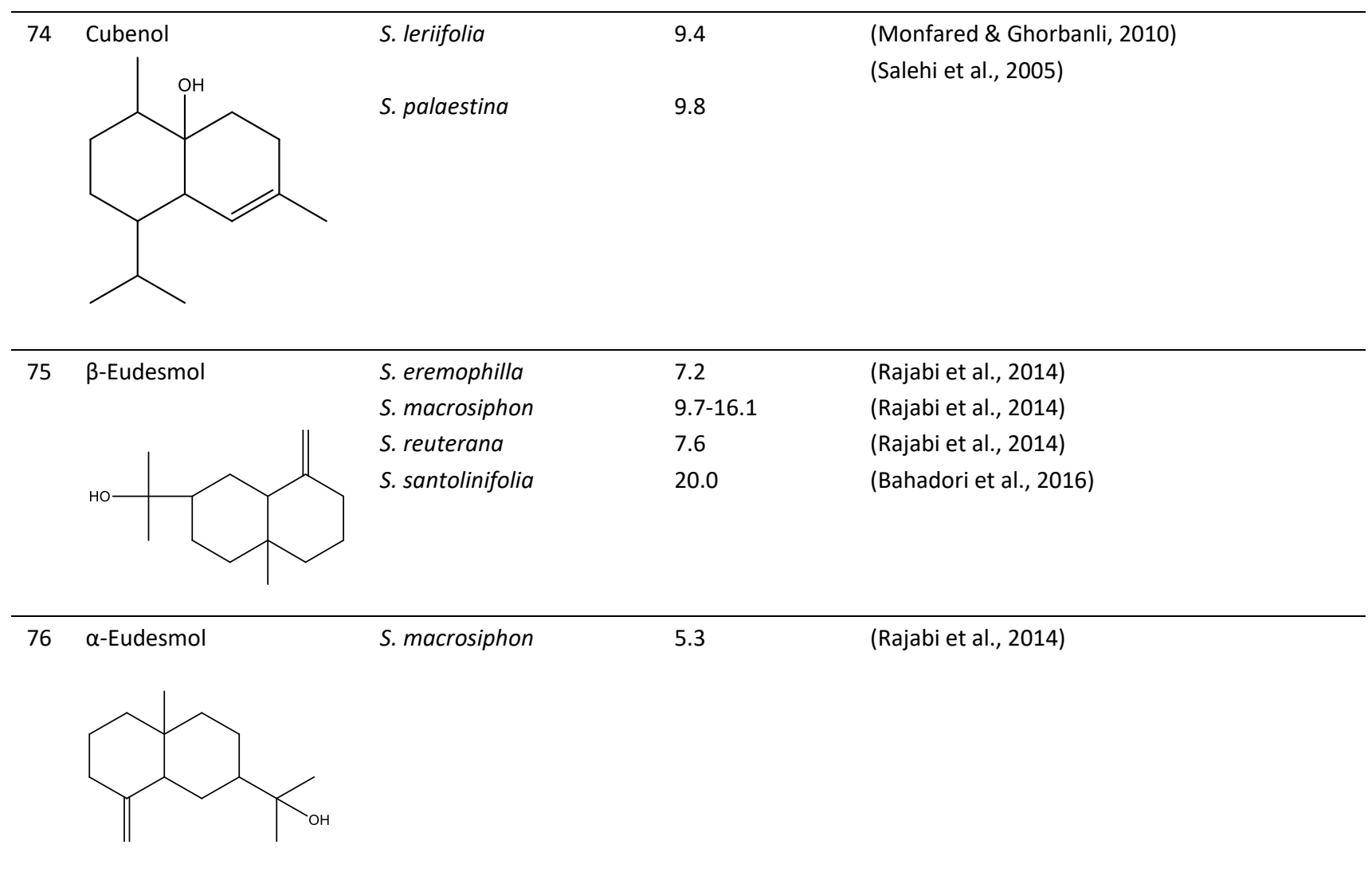




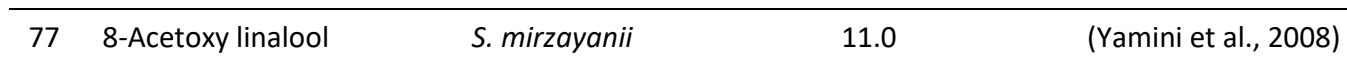

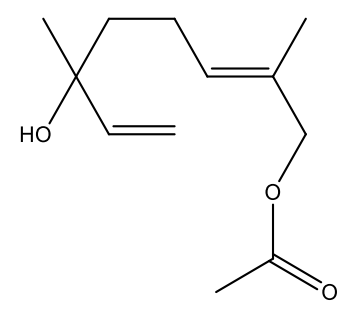

\begin{tabular}{lllll}
\hline 78 & 7-epi- $\alpha$-eudesmol & S. tebesana & 17.5 & (Goldansaz et al., 2017)
\end{tabular}<smiles>CC1=CCCC2(C)CCC(C(C)(C)O)CC12</smiles>

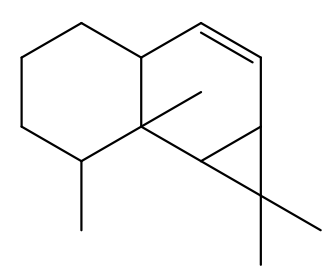<smiles>C=C(CCC=C(C)C)[C]1CC=C(C)CC1</smiles>

\begin{tabular}{lllll}
\hline 81 & Octadecane & S. sclareopsis & 20.6 & (Hemmati et al., 2018) \\
$\mathrm{C}_{18} \mathrm{H}_{38}$ & & &
\end{tabular}



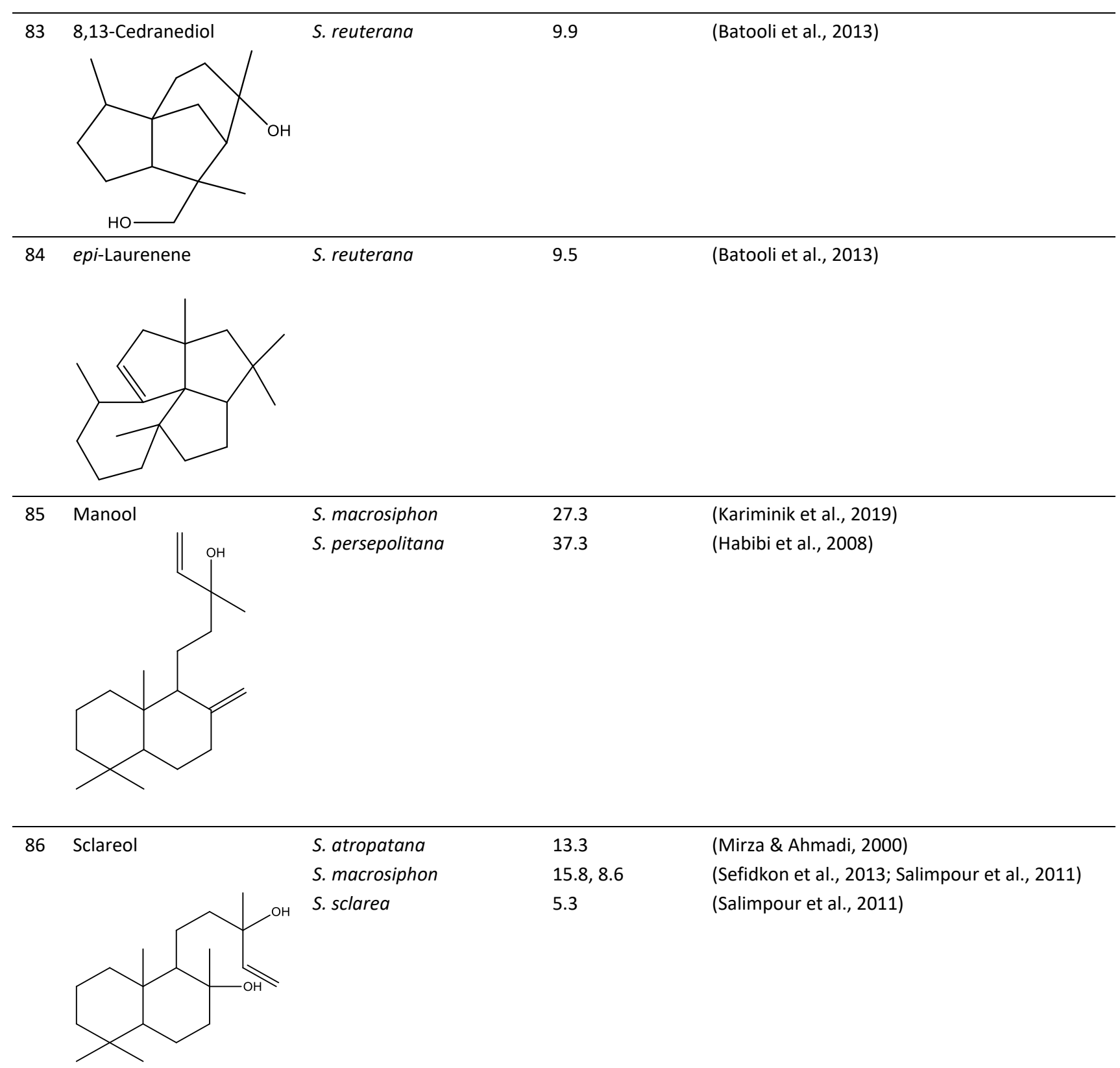

As shown in Table 2, 86 compounds were identified in the essential oil of Persian Salvia species in sizeable amounts (>5\%). The essential oil composition of these species is dominated by mostly the presence of monoterpene hydrocarbons, sesquiterpene hydrocarbons, oxygenated monoterpenes and oxygenated sesquiterpenes.

$\beta$-Caryophyllene as the most abundant component in many Salvia species essential oil, has been reported in S. aegyptiaca, S. aethiopis, S. aristata, S. atropatana, S. bracteata, S. chloroleuca, S. chorassanica, S. eremophilla, S. glutinosa, S. grossheimii, S. hydrangea, S. hypoleuca, S. limbata, S. macrochlamys, S. macrosiphon, S. multicaulis, S. nemorosa, S. palaestina, S. reuteriana, S. sclarea, S. sclareoides, S. sharifii, S. spinosa, S. tebesana, S. verticillata, S. virgata, S. xanthocheila of which the oils of S. bracteata, S. nemorosa and S. virgata contain more than $40 \% \beta$-caryophyllene. Germacrene D is the other abundant component reported in the most Salvia species such as S. aethiopis, S. atropatana, S. chloroleuca, S. glutinosa, S. grossheimii, S. hypoleuca, S. indica, S. limbata, S. macrosiphon, S. nemorosa, S. palaestina, S. reuteriana, S. sahendica, S. sclarea, S. sclareoides, S. sharifii, S. staminea, S. syriaca, S. urumiensis, S. verticillata, S. virgata, 
S. xanthocheila of which the S. aethiopsis, S. grossheimii and S. xanthocheila produce essential oils contained more than $40 \%$ of germacrene D.

$\alpha$-Pinene as the dominant component of the first category of the Iranian sage presents in 18 Salvia species including S. aegyptiaca, S. aristata, S. bazmanica, S. bracteata, S. chloroleuca, S. eremophila, S. hydrangea, S. hypoleuca, S. indica, S. leriifolia, S. limbata, S. macilenta, S. multicaulis, S. pachystachys, S. sahendica, S. santolinifolia, S. tebesana and $S$. urumiensis among which the $S$. macilenta and $S$. santolinifolia possess the essential oil with more than $60 \%$ of $\alpha$-pinene.

\section{Conclusion}

Data in the Table 2 represents a complex composition of the essential oils, especially for the same species. Indeed, the composition of essential oils depends on climatic and ecological conditions, plant organ and vegetative cycle stage. Thus, it is of utmost importance to characterize the essential oils composition as well as the influence of the referred parameters on its quality, in order to obtain essential oils of constant composition. This could only be possible if essential oils are extracted under the same conditions from the same organ of the plant which has been growing on the same soil, under the same climate and has been picked in the same season. It was also concluded that the most of the studied Iranian Salvia species were considered as sesquiterpene chemotype and $\beta$-caryophyllene followed by germacrene $D$, spathulenol and caryophyllene oxide were the most common and abundant in Persian Salvia species.

\section{ACKNOWLEDGMENT}

Supports from Tehran Medical Sciences, Islamic Azad University is gratefully acknowledged.

\section{CONFLICTS OF INTEREST}

The author does not have conflicts of interest to declare.

\section{REFERENCES}

Ahmadi, L., Mirza, M. (1999). Essential oil of Salvia multicaulis Vahl from Iran. Journal of Essential Oil Research, 11(3), 289-290.

Akhgar, M.R., Moradalizadeh, M., Faghihi Zarandi, A., Khorasanipour, M. (2011). Chemical composition of the essential oil from the aerial parts of Salvia bazmanica Rech. F. \& Esfand. Iranian Journal of Medicinal Aromatic Plants, 26(4), 555560.

Alizadeh, A., Shaabani, M. (2012). Essential oil composition, phenolic content, antioxidant and antimicrobial activity in Salvia officinalis L. cultivated in Iran. Advances in Environmental Biology, 6(1), 221-226.

Amabeoku, G.J., Eagles, P., Scott, G., Mayeng, I., Springfield, E. (2001). Analgesic and antipyretic effects of Dodonaea angustifolia and Salvia africana-lutea. Journal of Etnopharmacology, 75, 117-124.

Amiri, H. (2007). Quantative and qualative changes of essential oil of Salvia bracteata Bank et Sol. in different growth stages. DARU, 15(2), 79-82.

Asadipour, A., Mehrabani, M., Moghaddasian, M., Saber Amoli, S. (2013). Composition of the volatile oil of Salvia mirzayanii Rech. \& Esphand from Iran. Journal of Essential Oil Bearing Plants, 7(2), 182-185.

Asgarpanah, J., Oveyli, E., Alidoust, S. (2017). Volatile components of the endemic species Salvia sharifii Rech. F. \& Esfand., Journal of Essential Oil Bearing Plants, 20(2), 578-582.

Attaran, S., Abrishamchi, P., Assili, J. (2016). Essential oil (EO) composition and antioxidant activity of two Salvia leriifolia Benth. (Lamiaceae) populations from Iran. Nova Biologica Reperta, 3(2), 108-117. 
Bahadori, M.B., Valizadeh, H., Moridi Farimani, M. (2016). Chemical composition and antimicrobial activity of the volatile oil of Salvia santolinifolia Boiss. from Southeast of Iran. Pharmaceutical Sciences, 22, 42-48.

Bahernik, Z., Mirza, M. (2005). Volatile constituents of Salvia spinosa L. from Iran. Flavour and Fragrance Journal, 20(3), 311-312.

Bahernik, Z., Mirza, M. (2010). Identification of the essential oil of Salvia indica L. Journal of Essential Oil Bearing Plants, 13(4), 451-454.

Baldwin, I.T. (2010). Plant volatiles. Current Biology, 20(9), 392-397.

Batooli, H., Safaei-Ghomi, J., Haghir-Ebrahim-Abadi, A., Masoomi, R. (2013). Evaluating the chemical composition of the essential oil obtained from the vegetative and reproductive organs and an antimicrobial activity of essential oil and extract of two Salvia species in Kashan region, Journal of Kashan University of Medical Sciences, 16(6), 536-545.

Claßen-Bockhoff, R. (2017). Stamen construction, development and evolution in Salvia s.I. Natural Volatiles and Essential Oils, 4 (4) , 28-48.

de Almeida, R.N., de Fátima Agra, M., Souto Maior, F.N., de Sousa, D.P. (2011). Essential oils and their constituents: Anticonvulsant activity, Molecules, 16, 2726-2742.

Degenhardt, J., Kçllner, T.G., Gershenzon, J. (2009). Monoterpene and sesquiterpene synthases and the origin of terpene skeletal diversity in plants. Phytochemistry, 70(15-16), 1621-1637.

Dewick, P.M. (1997). Medicinal Natural Products, A Biosynthetic Approach. West Sussex, John Wiley \& Sons Publisher.

Ebrahimi, Z., Motavalizadeh Kakhky, A., Golshani, E., Ghabdian, M. (2014). Chemical composition and antimicrobial activity of essential oils of stem and hexane extract of aerial part of Salvia Chorassanica Bunge. The 22nd Iranian Seminar of Organic Chemistry, Faculty of Chemistry, University of Tabriz, Tabriz, Iran.

Ebrahimi, M., Ranjbar, S. (2016). Essential oils of Salvia hydrangea DC. ex Benth. from Kiasar-Hezarjarib regions, Iranimpact of environmental factors as quality determinants. Journal of Medicinal Plants and By-Products, 5(2), $159-167$.

El-Sayed, N.H., El-Eraky, W., Ibrahim, M.T., Mabry, T.J. (2006). Antiinflammatory and ulcerogenic activities of Salvia triloba extracts. Fitoterapia, 77, 333-335.

Emadipoor, E., Jamzad, M., Ghaffari, K., Ghadami, B., Jamzad, Z. (2016). Essential oil composition, total phenolic and favonoid contents, and biological activities of Salvia aristata Aucher ex Benth. Extracts. Journal of Essential Oil Bearing Plants, 19(6), 1426-1434.

Firdous, S., Dadass, A, K., Khan, K.M., Usmani, S.B., Ahmad, V.U. (1999). A new triterpenoid from the leaves of Salvia triloba. Fitoterapia, 70, 326.

Goldansaz, S.M., Hakimi Meybodi, M.H., Mirhosseini, A., Mirjalili, M.H. (2017). Essential oil composition of Salvia tebesana Bunge (Lamiaceae) from Iran. Records of Natural Products, 11(3), 310-314.

Guy, P., Kamatou, P., Viljoen, A.M., Steenkamp, P. (2010). Antioxidant, antiinflammatory activities and HPLC analysis of South African Salvia species. Food Chemistry, 119, 684-688.

Habibi, Z., Yousefi, M., Aghaie, H.R., Rustaiyan, A. (2008). Chemical composition of essential oil of Salvia persepolitana Boiss. and Salvia rhytidea Benth. from Iran. Journal of Essential Oil Research, 20(1), 1-3.

Haghighat, M.H., Alizadeh, A., Nouroznejadfard, M.J. (2012). Essential oil composition and antimicrobial activity in Iranian Salvia mirzayanii Rech. \& Esfand. Advances in Environmental Biology, 6(7), 1985-1989.

Hemmati, P., Ghavyar, H., Amiri, H. (2018). Chemical composition of essential oil and antioxidant activity of Salvia sclareopsis an endemic species from Iran. Journal of Essential Oil Bearing Plants, 21(4), 1138-1145. 
Jamzad, M., Rustaiyan, A., Jamzad, Z., Masoudi, S. (2011). Essential oil composition of Salvia indica L., Thymus caucasicus Wind. Ex Ronniger subsp. grossheimii (Ronniger) Jalas. and Ballota nigra L. Three Labiatae species from Iran. Journal of Essential Oil Bearing Plants, 14(1), 76-83.

Jamzad, M., Shahbeig, M., Jamzad, Z., Yari, M., Ghadami, B. (2012). Volatile constituents of two Labiateae species from Iran and antibacterial activity of the acetone extract of them. Journal of Essential Oil bearing plants, 15(3), 380-386.

Jassbi, A.R., Asadollahi, M., Masroor, M., Schuman, M.C., Mehdizadeh, Z., Soleimani, M., Miri, R. (2012). Chemical classification of the essential oils of the Iranian Salvia species in comparison with their botanical taxonomy. Chemistry and Biodiversity, 9, 1254-1271.

Javidnia, K., Miri, R., Kamalinejad, M., Nasiri, A. (2002). Composition of the essential oil of Salvia mirzayanii Rech. f. \& Esfand from Iran. Flavour and Fragrance Journal, 17(6), 465- 467.

Kabouche, A., Kabouche, A., Öztürk, M., Kolak, U., Topçu, G. (2007). Antioxidant abieetane diterpenoids from Salvia barrelieri. Food Chemistry, 102, 1281-1287.

Kariminik, A., Moradalizadeh, M., Foroughi, M.M., Tebyanian, H., Motaghi, M.M. (2019). Chemical composition and antibacterial activity of the essential oils extracted from 4 medicinal plants (Labiatae) of Kerman, Iran. Journal of Applied Biotechnology Reports, 6(4), 172-179.

Kazemi, M. (2015). Essential oil of the aerial parts of Salvia officinalis (Lamiaceae) from Iran. Journal of Essential Oil Bearing Plants, 18(3), 725-727.

Kazemizadeh, Z., Habibi, Z., Yousefzadi, M., Ashabi, M.A., Heydari Rikan, M. (2010). Chemical composition and antibacterial activity of the essential oil of Salvia macrochlamys Boiss. \& Kotschy. from west Azarbayejan Province. Journal of Medicinal Plants, 9(33), 75-82.

Khaliazadeh, M.A., Esmaeili, A., Rustaiyan, A., Masoudi, S. (2011). Chemical composition of essential oils of three Salvia species growing wild in Iran. Chemistry of Natural Compounds, 46(6), 985-987.

Kusumi, T., Ooi, T., Hayashi, T., Kakisawa, H. (1985). A diterpenoid phenalenone from Salvia Miltiorrhiza, Phytochemistry, 24, 2118-2120.

Lari Yazdi, H., Goudarzi, M., Yazdani, D., Chehregai, A.K. (2005). Essential oils composition of leaves and flowers of Salvia syriaca L. and S. reuterana Boiss. from Borujerd-Iran. Journal of Medicinal Plants. 4(16), 15-21.

Lu, Y., Foo, L.Y. (2002). Polyphenolics of Salvia. A review. Phytochemistry, 59, 114-140.

Maklad, Y.A., Aboutabl, E.A., El-Sherei, M.M., Meselhy, K.M. (1999). Bioactivity studies of Salvia transsylvanica (Schur ex Griseb) grown in Egypt. Phytotherapy Research, 13, 147-150.

Mirza, M., Ahmadi, L. (2000). Composition of the essential oil of Salvia atropatana Bunge. Journal of Essential Oil Research, 12(5), 575-576.

Mirza, M., Bahernik, Z. (2007). Extraction and identification of chemical components of the essential oil of Salvia compressa Vent. Iranian Journal of Medicinal Aromatic Plants, 22, 431-436.

Mirza, M., Bahernik, Z, (2010). Chemical composition of the essential oil of Salvia grossheimii from Iran. Chemistry of Natural Products, 46(5), 822-823.

Mirza, M., Bahernik, Z., Jamzad, Z. (2003). The extraction and identification of the essential oil constituents of Salvia mirzayanii Rech. F. \& Esfand. Iranian Journal of Medicinal Aromatic Plants, 19(2), 117-124.

Mirza, M., Mozaffarian, V., Bahernik, Z. (2005). Composition of the essential oil of Salvia limbata CA Mey. Journal of Essential Oil Research, 17(1), 10-11.

Mirza, M., Sefidkon, F. (1999). Essential oil composition of two Salvia species from Iran, Salvia nemorosa L. and Salvia reuterana Boiss. Flavour and Fragrance Journal, 14(4), 230-232. 
Monfared, A., Ghorbanli, M. (2010). Composition of the essential oils of Salvia leriifolia Benth. growing wild in around of two mines in Iran. Research Journal of Phytochemistry, 4(1), 13-17.

Morteza-Semnani, K., Saeedi, M., Akbarzadeh, M. (2014). Chemical composition of the essential oil of Salvia limbata C. A. Mey. Journal of Essential Oil Bearing Plants, 17(4), 623-628.

Mozaffarian, V. (1996). Dictionary of Iranian plant names. Farhang Moaser Publisher.

Nickavar, B., Mojab, F., Asgarpanah, J. (2005). Volatile composition of the essential oil of Salvia hypoleuca Benth., International Journal of Aromatherapy, 15(1), 51-53.

Nieto, M., García, E.E., Giordano, O.S., Tonn, C.E. (2000). Icetexane and abietane diterpenoids from Salvia gilliessi. Phytochemistry, 53, 911-915.

Norouzi-Arasi, H., Yavari, I., Chalabian, F., Baghaii, P., Kiarostami, V., Nasrabadi, M., Aminkhani, A. (2005). Volatile constituents and antimicrobial activities of Salvia suffruticosa Montbr. \& Auch. ex Benth. from Iran. Flavour and Fragrance Journal, 20, 633-636.

Rajabi, Z., Ebrahimi, M., Farajpour, M., Mirza, M., Ramshini, H. (2014). Compositions and yield variation of essential oils among and within nine Salvia species from various areas of Iran. Industrial Crops and Products, 61, 233-239.

Rauter, A.P., Branco, I., Lopes, R.G., Justino, J., Silva, V.M.F., Noronha, J.P., Cabrita, E.J., Brouard, I., Bermejo, J. (2007). A New lupenetriterpenetriol and anticholinesterase activity of Salvia sclareoides. Fitoterapia, 78, 474-481.

Rustaiyan, A., Akhgar, M.R., Masoudi, S., Nematollahi, F. (2005). Chemical composition of essential oils of three Salvia species growing wild in Iran: Salvia rhytidea Benth., S. limbata C.A. Mey. and S. palaestina Benth. Journal of Essential Oil Research, 17(5), 522-524.

Sajjadi, S.E., Ghannadi, A. (2005). Essential oil of the Persian sage, Salvia rhytidea Benth. Acta Pharmaceutica, 55, 321326.

Sajjadi, S.E., Shahpiri, Z. (2004). Chemical composition of the essential oil of Salvia limbata C. A. Mey. DARU, 12(3), 9497.

Salehi, P., Bazzaz Tolami, L., Sefidkon, F. (2005). Essential oil composition of Salvia xanthocheila Boiss. ex Benth. from Iran. Journal of Essential Oil Research, 17(4), 442-443.

Salehi, P., Sefidkon, F., Tolami, L., Sonboli, A. (2005). Essential oil composition of Salvia palaestina Benth. from Iran. Flavour and Fragrance Journal, 20(5), 525-527.

Salehi, P., Sonboli, A., Moghadam, S. (2013). Essential oil composition and antioxidant activity of Salvia staminea Benth. extracts. Journal of Essential Oil Bearing Plants, 16(5), 582-587.

Salehi, P., Sonboli, A., Nejad Ebrahimi, S., Yousefzadi, M. (2004). Essential oil composition and antimicrobial activity of Salvia sahendica Boiss \& Buhse. in different phenological stage. Iranian Journal of Pharmaceutical Research, 2, 91.

Salehi, S., Golparvar, A.R., Hadipanah, A. (2014). Chemical composition of essential oil from the aerial parts of Salvia spinosa L. collected from Isfahan. Journal of Herbal Drugs, 5(2), 107-112.

Salimpour, F., Mazooji, A., Akhoondi Darzikolaei, S. (2011). Chemotaxonomy of six Salvia species using essential oil composition markers. Journal of Medicinal Plants Research, 5(9), 1795-1805.

Sefidkon, F., Hooshidary, Z., Jamzad, Z. (2007). Chemical variation in the essential oil of Salvia bracteata Banks \& Soland from Iran. Journal of Essential Oil Bearing Plants, 10(4), 265-272.

Sefidkon, F., Mirza, M. (1999). Chemical composition of the essential oils of two Salvia species from Iran, Salvia virgata Jacq. and Salvia syriaca L. Flavour and Fragrance Journal, 14(1), 45-46.

Sefidkon, F., Mirza, M., Javidtash, I. (2013). Essential oil composition of Salvia macrosiphon Boiss. from Iran. Journal of essential Oil Bearing Plants. 8(2), 126-129. 
Sepahvand, R., Delfan, B., Ghanbarzadeh, S., Rashidipour, M., Veiskarami, G., Ghasemian-Yadegari, J. (2014). Chemical composition, antioxidant activity and antibacterial effect of essential oil of the aerial parts of Salvia sclareoides, Asian Pacific Journal of Tropical Medicine, 7S1, S491-496.

Shakeri, A., Sharifi, M.J., Fazly Bazzaz, B.S., Asili, J. (2018). Bioautography detection of antimicrobial compoundsfrom the essential oil of Salvia pachystachys. Current Bioactive Compounds, 14(1), 80-85.

Sonboli, A., Babakhani, B., Mehrabian, A.R. (2006). Antimicrobial activity of six constituents of essential oil from Salvia. Zeitschrift fur Naturforschung C, 61 c, 160-164.

Sonboli, A., Fakhari Zavareh, A., Sefidkon, F. (2005). Chemical composition of the essential oil of Salvia macilenta from Iran. Chemistry of Natural compounds, 41(2), 168-170.

Sonboli, A., Salehi, P., Gharehnaghadeh, S. (2016). Chemical variability in the essential oil composition of Salvia hypoleuca, an endemic species from Iran. Journal of Essential Oil Research, 28(5), 1-7.

Tavassoli, A., Esmaeili, A., Ebrahimzadeh, M.A., Safaeyan, S., Akbarzade, M., Rustaiyan, A. (2009). Chemical composition of essential oil and antibacterial activity of Salvia glutinosa L. growing wild in Iran. Journal of Applied Chemical Research, $3(10), 1-10$.

Tepe, B., Daferera, D., Sokmen, A., Sokmen, M., Polissiou, M. (2005). Antimicrobial and antioxidant activities of the essential oil and various extracts of Salvia tomentosa Miller (Lamiaceae). Food Chemistry, 90, 333-340.

Tepe, B., Dönmez, E., Unlu, M., Candan, F., Dimitra, D., Vardar-Unlu, G. (2004). Antimicrobial and antioxidative activities of the essential oils and methanol extracts of Salvia cryptantha (Montbret et Aucher ex Benth.) and Salvia multicaulis (Vahl). Food Chemistry, 84, 519-525.

Usama, W.H., Mohamed, A.E. (2006). Flavonoids and antimicrobial activity of Salvia lanigera Poir. Journal of the Arab Society for Medical Research, 1, 159-167.

Walker, J.B., Sytsma, K.J., Treutlein, J., Wink, M. (2004). Salvia (Lamiaceae) is not monophyletic: implications for the systematics, radiation, and ecological specializations of Salvia and tribe Mentheae. American Journal of Botany, 91, 1115-1125.

Yamini, Y., Khajeh, M., Ghasemi, E., Mirza, M., Javidnia, K. (2008). Comparison of essential oil compositions of Salvia mirzayanii obtained by supercritical carbon dioxide extraction and hydrodistillation methods. Food Chemistry, 108(1), 341-346.

Yousefi, M., Nazeri, V., Mirza, M. (2015). Effects of environmental conditions on the quantity and quality of Salvia leriifolia Benth. essential oil. Iranian Journal of Medicinal Aromatic Plants, 30(6), 861-877.

Zare, S., Jassbi, A.R. (2014). Using chemical classification of the essential oils to differentiate Salvia sharifii from S. macrosiphon, Journal of Essential Oil Bearing Plants, 17(6), 1356-1360. 